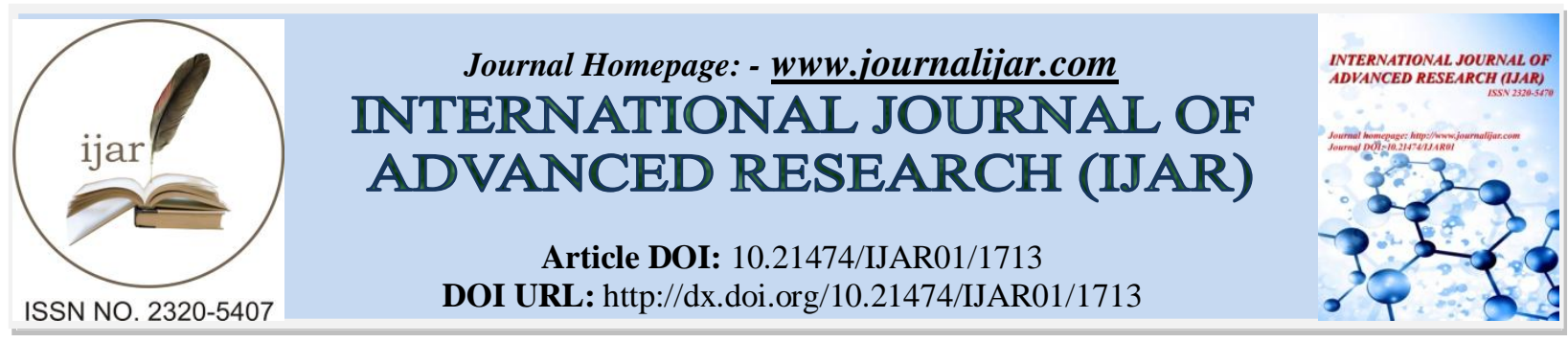

RESEARCH ARTICLE

\title{
ALPINE AND SUBALPINE FLORA OF SHARR MOUNTAIN - (MACEDONIAN PART).
}

\section{Nasuf Abdii ${ }^{1}$ and Murat Xhulaj'.}

1. University of Tetova, Department of Biology, Macedonia.

2. University of Tirana, Department of Biology, Albania.

\section{Manuscript Info}

Manuscript History

Received: 12 July 2016

Final Accepted: 19 August 2016

Published: September 2016

Key words:-

flora, herbarium, biotope, family, species.

\begin{abstract}
In this scientific work will be presented partial data of four year scientific researcher work $(2013,2014,2015,2016)$, a phase which coincides with different phases of vegetation. This study has been done for the first time, especially about the Flora of Sharr Mountain. The study is concentrated in 20 station in the Sharr Mountain. The accumulation of the scientific material was conducted from early spring until late autumn, by preparing herbarium, accompanied by data for site-collection, date, biotope etc. During this study a rich material has been collected, of about 640 copies. From the previous floristic analysis so far, it results that the Flora of the Sharr Mountain is rich in types. The selected material consists of 58 families, 167 genders and 388 species.
\end{abstract}

Copy Right, IJAR, 2016,. All rights reserved.

\section{Introduction:-}

The subalpine zone in Sharr Mountain - stretching from 1700 - $2100 \mathrm{~m}$ (rare $2200 \mathrm{~m}$.) above sea level. There we can find pine forests or molika (Pinus peuce) a spruce (Pinus abies) and pines (Pinus mugo) and communities of Bruckenthalia spiculifolia, Vaccinium myrtyllus, etc.

Sub alpine and alpine communities - stretching above the upper forest belt from 1600-2770 m., where climatic conditions are harsh (long winters, short summers and short vegetation period). Here are developed different communities in quite heterogeneous substrate (acidic soil, limestone substrate, mountain brooks, rocky substrate, etc.).

In Sharr Mountain are developed other types of vegetation community, such as the clearing of forest communities, ruderale communities, violated surfaces communities, communities of shrubs, etc.

Sharr Mountain is one of the most important Balkan and European centers and of most qualitative endemism including relicts, endemo and endemic relicts and types. In the Sharr Mountain there are around 200 taconic, endemic and sub-endemic plants, (species, sub-species and varieties), steno-endemic orofits (mountain species). These species are of tertian age and rarely from glacial. Such species require special care: Silene schmuckeri, Dianthus scardicus, Bornmullera dieckii, Draba korabensis, Sedum flexuosum, Potentilla doerfleri, Crocus scardicus and Oxytropis korabensis.

Tertian orofits (mountainous species of tertian age). Most of them are steno-endemic and sub-endemic species: Pinus peuce, Pinus heldreichii, Silene Waldsteinii, Silene lerchenfeldiana, Silene asterias, Ptilotrichum rupestre, 
Shievereckia doerfleri, Iberis sempervirens, Hesperis Dinarica, Saxifraga Androsaceae, Saxifraga glabella, Potentilla montenegrina, Anthyllus aurea, Acer heldreichii, Rhamnus pumilla, Viola Grisebachiana, Heacleum orphanidis, Soldanella dimoniei, Veronica satureioides, Tozzia alpia, Ramonda serbica and Ramonda nathaliae, Nerthecium scardicum, Lilium albanicum, Gymnadenia frywaldsiana, Rhododendron ferrugineum and Linaria alpina.

Glacial species with arctic alpine spreading (forms from the ice age). They are very rare in the Balkan Peninsula: Selaginella Selaginoides, Diphasium alpinium, Cryptogramma crispa, Salix herbaceae, Salix reticulata, Rumex nivalis, Silene rupestris, Rhodiola rosea, Saxifraga bryoides, Saxifraga androsaceae, Geum reptans, Epilobium anagallidifolium, Loiseleuria procumbens, Veronica alpina, Veronica aphylla, Pedicularis oederi, Erigeron uniflorus, Saussurea alpina, Carex foetida [1].

\section{Material and metods:-}

\section{Geographical position of Sharr Mountain:-}

The geographical position of the massif of Shara is suitable and represents a sample of a mountain massif with clear morphoplastic differentiation. This mountain massif lies in the northwestern part of the Republic of Macedonia and is part of Polog region, ie it constitutes the western part [map. no. 1].

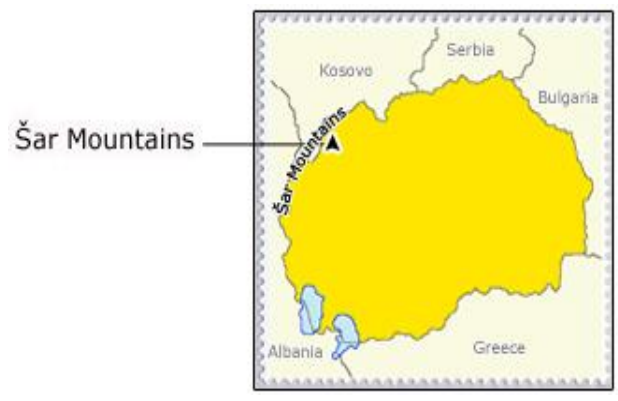

Location of the Sharr Mountain in R. Macedonian [Map no. 1].

The main elements of its geographical position are as follows: it is part of the Sharron-Pind mountain system, respectively it belongs to Dinarids; It is exposed to climatic influences of the Adriatic Sea and the Aegean Sea; located in the central part of the Balkan Peninsula.

The Sharr Mountain represents the largest mountain massif in Macedonia and lies in the geographical coordinates: between $42^{\circ} 41^{\prime} 43^{\prime \prime}$ and $42^{\circ} 16^{\prime} 34^{\prime \prime}$ geographical north latitude and between $20^{\circ} 34^{\prime} 51$ "and $21^{\circ} 16^{\prime} 00^{\prime \prime}$.

Unlike other mountains of the Balkan Peninsula that are stretched in northwest-southeast, Sharr has massive stretch in northeast-southwest. This mountain massif lies in the length of $80 \mathrm{~km}$ and a width of 10-20 km [2].

Geological aspects of the researched region

From the geological aspect (Менковић, 1978), in the creation of the mountains of the Sharr region, are schist rocks, but there is also limestone and magmatic rocks.

The magmatic rocks in the Sharr Mountain contain chrome, while in Bistra it contains iron. From non-metals there is a big surface of gypsum and qualitative marble surfaces near the town of Gostivar.

From the geological content aspect, dominate the Paleozoic plates mainly with a big quantity of lime and dolomite. The Bistra Mountain from the geological aspect consists of old Paleozoic rocks, with glacial relief as well, especially the circles [3].

\section{Climate conditions:-}

According to the geographic position (Rodić, 1987), the Republic of Macedonia is located in the Southern Europe with a meridian 41 and 42 passing through it in the northern geographic width, which means that it is closer to the Ecuador than Northern Pole. The geographic width affects directly in the intensity and extension of solar radiation 
and this is lowered from south to north. The solar radiation extension and intensity is affected by the nearness sea as well.

From this point of view, the territory of the Republic of Macedonia even though it doesn't have direct cruise in the sea, is under the influence of side seas, because from the Aegean Sea in the south it is about $60 \mathrm{~km}$ far away (airline), and from the Adriatic Sea in west around $80 \mathrm{~km}$. Generally it has Mediterranean mild climate. However, the influence of the Mediterranean climate does not enter in its depth, due to the high mountains in the west and south.

From the West there can be seen the influence of the Atlantic Ocean, although it is located very far. From there western winds blow bringing lots of air humidity during the whole year, and during the spring and autumn months, they bring rain. In the researched area, according to Lazarevski (Lazarevski, 2002) there are three main climate types:

Mountainous climate it is characteristic for mountains above $1000 \mathrm{~m}$ altitude. It has long and cold winters with snow and short and chilly summers. Here, the spring is colder than autumn. This climate is more present than the others, because the majority of the researched part of the region has high mountain terrains [4].

Table no. 1. Expedition realized

\begin{tabular}{|l|l|l|}
\hline No. & Date & Location \\
\hline 1. & 26.05 .2013 & Upper Palcist \\
\hline 2. & 16.06 .2013 & Lisec \\
\hline 3. & $26.05 ., 02.06 ., 07.07 ., 04.08 .2013$ & Sellca's baths \\
\hline 4. & 06.10 .2013 & Kodra e Diellit (Popova Sapka). \\
\hline 5. & 24.05 .2013 & Lajthiza (Leshnica) \\
\hline 6. & 01.10 .2013 & Maja e Diellit (Titov Vrv) \\
\hline 7. & 02.09 .2014 & Rogaçeva \\
\hline 8. & 10.10 .2014 & Luboten \\
\hline 9. & 11.11 .2014 & Veshalla \\
\hline 10. & 25.05 .2015 & Gajre \\
\hline 11. & 24.05 .2015 & Maja e Diellit (Titov Vrv) \\
\hline 12. & 06.06 .2015 & Teqe e Shipkovicës \\
\hline 13. & 07.06 .2015 & Jellovjan \\
\hline 14. & 23.06 .2015 & Jellak, Cerepashin \\
\hline 15. & 27.06 .2015 & Jellovjan- Mazraça- liqeni i Rakovecit \\
\hline 16. & 09.07 .2015 & v. Brest, three waters above village Tearce. \\
\hline 17. & 1.8 .2015 & mountain above the village Bozofc. \\
\hline 18. & 9.08 .2015 & Lakavica village, Sermnova (Gostivar). Altitude 780 m. \\
\hline 19. & 30.08 .2015 & Maja e Diellit (Titov Vrv) \\
\hline 20. & 12.09 .2015 & v. Varvara \\
\hline 21. & 24.05 .2016 & Bogovina's lacke \\
\hline 22. & 04.06 .2016 & Black lake \\
\hline 23. & 22.06 .2016 & Kodra e diellit, Maja e diellit (Popova Sapka, Titov vrv) \\
\hline 24. & 22.06 .2016 & Kodra e diellit, Maja e diellit (Popova sapka, Titov vrv) \\
\hline 25. & 10.07 .2016 & Xhinibeg \\
\hline & & \\
\hline
\end{tabular}

\section{Results of the study:-}

In publications of domestic and foreign authors the number of taxa described in Flora of Shara reaches at 590 taxa while in this scientific research the total number of plant species reaches up to 650, of which 381 taxa are found in the alpine and subalpine zone. Species of the family Compositae and class Monocotiledona that are in flora of Sharr Mountain are not addressed by domestic and foreign researchers and so during this scientific research total number of species in flora Sharr Mountain reaches up to 650. These data provide evidence for a rich flora of the massif of Sarr. 
By analyzing and comparing our current material with what is known to date for Sharr Mountain is observed pronounced similarity and large number of endemic plants.

The family of plants with larger number of taxa in the flora of Sharr Mountain is: Ranunculaceae, Caryophyllaceae, Cruciferae, Rosaceae, Leguminosae, Umbelliferae.

During the scientific research within the flora of Sharr Mountain were found about 20 plant species wich belongs to monocotyledonae ( seeds contain only one embryonic leaf, or cotyledon) and the material collected so far in Flora of Sharr Mountain noted significant number of mokotiledone plants, which belongs to 8 families, 16 genders. A significant number of monocotyledons species, are important for further studies in the Sharr Mountain vegetation.

\section{Tax treated hierarchy:-}

The following is a summarized ranking taxonomic [species of subalpine zone 1700- $2100 \mathrm{~m}$ rarely 2,200 m. and alpine zone of- $2770 \mathrm{~m}$.] which is followed in this study, and which is based on the nomenclature of Hill et al. (2006). Next to each species it is given the type of substrate and the altitude at which certain species is collected $[6,7,8,9]$.

\section{Lycopodiaceae}

1. Huperzia Bernh. 1. Huperzia Selago (L).; 2. Diphasium C. Presl. 2. Diphasium alpinum L. [Silicate substrate, Sharr Mountain].

2. Ophyoglossaceae

3. Botrychium L. Swartz.3. Botrychium lunaria [Mountain meadows and pastures. 1000-2650 m.].

3. Cryptogrammaceae

4. Cryptogramma R.Bc. Sh 4. Cryptogramma crispa L. [Stony silicate for 1800-2400 m.].

4. Aspleniaceae

5. Asplenium L. 5. Asplenium viride. [Silicate rocks crevices. 1500-2600 m.]; 6. Asplenium ruta-muraria [Limestone rock's crevices. $2300 \mathrm{~m}$.].

5. Athyriaceae

6. Athyrium Roth. 7. Athyrium filix-femina. [Wet alpes, area alpines $2200 \mathrm{~m}$.].

6. Pinaceae

7. Abies. Miller. 8. Abies borisi-regis. [1000-1800 m.].

8. Picea. A. Dietr. 9. Picea abies [Vraca. 1400-2100 m.].

9. Pinus L. 10. Pinus nigra [100-1800 m.]. 11. Pinus sylvestris [1000-1800 m.]. 12. Pinus peuce Grisebach [Usually grows on silicate, but can be seen on limestone too; serpentine 1400-2200 m.].

\section{Cupresaceae}

10. Juniperus L. 13. Juniperus communis [Meadows, scrubs and forest]; 14. Juniperus nana [area Alps 1600-1900 m.].

\section{Taxaceae}

11. Taxus L. 15. Taxus bacata [fores with fagus and abies].

\section{Ranunculaceae}

12. Trollius L. 16. Trollius europaeus L. [pastures and meadows in wet area alpines].

13. Actaea L. 17. Actaea spicata [forest with fagus].

14. Caltha L. 18. Caltha palustris [wet meadows; wetlands; along streams].

15. Aconithum L. 19. Aconitum lamarckivar. Macedonicum [alpine pastures].

16. Anemone L. 20. Anemone nemorosa [Mainly in deciduous forests and near streams]. 21. Anemona narcisiflora [Alpine pastures].

17. Clematis L. 22. Clematis vitalba [grows in riparian forests, along water flows, mainly in the range from lowlands to foothils].

18. Ranunculus L. 23. Ranunculus repens [wet meadows, weatlands, along streams]; 24. Ranunculus acris L. [wet places in forests]; 25. Ranunculus serbicus Vis. [Wet meadows, weatlands, along streams]; 26. Ranunculus illyricus [Në zonën e pyjeve me ah në vende të thata dhe kullosa]. 27. Ranunculus degeni [Wet meadows, weatlands, along streams]; 28. Ranunculus crenatus Waldst. [Wet meadows and stony]; 29. Ranunculus platanifolius L. [Wet meadows, weatlands, along streams, in the of beach, fir-beach]; 30. Ranunculus montenegrinus (Hal.) Lindt. [Maja e Diellit $2450 \mathrm{~m}$. (Titov Vrv)].

19. Thalictrium L. 31. Thalictrium alpinum L. [alpine pastures].

10. Fumariaceae

20. Corydalis Vent 32. Coridalis solida [alpine pastures 1700-2700 m.]. 


\section{Urticaceae}

21. Urtica L. 33. Urtica dioica [Near the house, woods. 2000m. highest. 34. Urtica parietaria $2000 \mathrm{~m}$. highest).

12. Fagaceae

22. Fagus L. 35. Fagus sylvatica [to 800-2000 m. highest].

13. Betullaceae

23. Betula L. 36. Betula pendula [Street Lisec- Kodra e diellit (Popova Sapka) 300-1900m highest].

24. Corylus 37. Corylus avellana [in the scrub forests $1200-1700 \mathrm{~m}$.].

14. Caryophyllace

25. Arenaria L. 38. Arenaria biflora [alpine pastures. 1400-2400 m.].

26. Minuartia L. 39. Minuartia setaceae Thuill. [Në gurishta gëlqerore, vende gurërore, nga ultësira deri $2000 \mathrm{~m}$.]; 40. Minuartia recurve [Në kullosa të zonës alpike dhe vende me gurërore. 1700-2750 m.].

27. Stellaria L. 41. Stellaria nemorum [Wet meadows, weatlands, along streams 800-2000 m.]; 42 . Stellaria alsine [Wet meadows, weatlands, along streams 1200-2000 m.]; 43. Stellaria graminea [alpine pastures. 2200 m.].

28. Cerastium L. 44. Cerastium lanatum [Area alpine . From 1300-2650 m.].

29. Moenchia Ehrh. 45. Moenchia mantica L. [pastures from 1800 m.].

30. Sagina L. 46. Sagina subulata (Sëartz). [alpine pastures and limestone screes from 1800 to 2300 m.]. 47 . Sagina procumbens L. [wet areas to $2000 \mathrm{~m}$.].

31. Scleranthus L.48. Scleranthus perennis L. [Në gurishta, kullosa të zonës alpike, përreth rrugëve deri $1800 \mathrm{~m}$.]. 49. Scleranthus annuus L. [2000 m.].

32. Paronychia Miller 50. Paronychia chionaea [on limestone rocky, for 750-2500 m.].51. Paronichia albanica [limestone rocky. Luboten $2500 \mathrm{~m}$.].

33. Herniaria L. 52. Herniaria glabra [pastures, to $2100 \mathrm{~m}$.].

34. Spergularia (Pers.) 53. Spergularia rubra [rocky and alpine pastures, to $2200 \mathrm{~m}$.].

35. Lychnis L. 54. Lychnis viscaria [limestone rocky $2500 \mathrm{~m}$.].

36. Silene L.55. Silene bupleroides [pastures and rocky places, for 500-2500 m.]; 56. Silene sendtneri [pastures, for 1500-2500 m.]; 57. Silene multicaulis Guss. limestone rocky and rocky places, for 1300-2300 m.]; 58. Silene waldsteini Griseb. [Silicate rocky, pastures and rocky places, for 1700-2200 m.]; 59. Silene ciliate Pourret. [on limestone rocky and pastures, for 1000-2300 m.]; 60. Silene acaulis L. [on limestone rocky and pastures $2000-2700$ m.]; 61. Silene lerchenfeldiana Baumg. [Silicate rocky crevices, for 800-2100 m.]; 62. Silene heuffelii Soo. Feddes Repert. [Pyje me ah, area alpine and subalpines].

37. Drypis L. 63. Dripis spinosa L. [on limestone rocky, for 1400-2500 m.].

38. Saponaria L. 64. Saponaria bellidifolia Sm. [on limestone rocky and pastures, for 500-2000 m].

39. Dianthus L. 65. Dianthus scardicus Wettst. [Endemic, Luboten, for 1900-2700 m.]; 66. Dianthus minutiflorus (Borbas) [1500-2500 m.]; 67. Dianthus superbus Ellak [endemic, 2000 m.]; 68. Dianthus deltoids L. [Luboten]; 69. Dianthus degeni Bald. [for 1300-2700 m]; 70. Dianthus cruentus Griseb., [900-2500 m]; 71. Dianthus hyalolepis Acht. Et Lindtner. [Kodra e diellit-Leshnica 1650 m.].

15. Polygonaceae

40. Polygonum L 72. Polygonum arenastrum [Tetovo to $1650 \mathrm{~m}$.]; 73. Polygonum bistoria [Sharr Mountain 1600 m.]; 74. Polygonum alpinum [Sharr mountain-Vraca, area subalpine].

41. Oxiria Hill 75. Oxiria digyna [Sharr Mountain, Livadica].

42. Rumex L. 76. Rumex acetosella [Sharr Mountain]; 77. Rumex scutatus [Sharr Mountain 2000 m.]. 78. Rumex nivalis [2000-2650 m.]; 79. Rumex arifolius [deri 2000 m.]; 80. Rumex alpinus L. [1.500-2.450]; 81. Rumex acetosa [2200 m.].

16. Plumbaginaceae

43. Armeria Willd. 82. Armeria canescens [Cerepashina, Maja e Diellit (Titov vrv), Luboten, 1.500-2.500 m.]; 83. Armeria rumelica [deri $2.200 \mathrm{~m}$.].

17. Hypericaceae

44. Hypericum L. 84. Hypericum barbatum [2100 m.]; 85. Hypericum maculatum [Popova Sapka (Popova Shapka), Cerepashina, $2200 \mathrm{~m}$.]; 86. Hypericum perforatum [2000 m.].

18. Violaceae

45. Viola L. 87. Viola odorata [1.600 m.]; 88.Viola reichenbachiana Jordan ex Boreau [2.000 m.]; 89. Viola grisebachiana [2.500 m.]; 90. Viola ivonis [1.800-2.100 m.]; 91. Viola chelmea [Cerepashina, Maja e Diellit (Titov Vrv), Luboten, 2100-2500 m.]; 92. Viola schariensis [1700- 2400 m.]; 93. Viola elegantula [1200-2000 m.]; 94. Viola latisepala [1200-2400 m.]; 95. Viola macedonica [800-2000 m.]; 96. Viola orphanidis Boiss.[1.300- 2000 m.]; 97. Viola gracilis Sibth [1.300-2.050 m.]; 98. Viola kosanini (Degen) Hayek. [700-2.100 m.].

19. Cistaceae 
46. Helianthemum Miller. 99. Helianthemum nummularium [2.500 m.]; 100. Heilianthemum oelandicum (L.); 101. Heilianthemum canum (L.) [ area alpine].

20. Cruciferae

47. Arabidopsis (DC) Heynh 102. Arabidopsis thaliana [1700 m.].

48. Erysimum L. 103. Erysimum comatum [2100 m.]; 104. Erysimum korabense Kumm. [bimë e zonës alpike]; 105. Erysimum kuemmerlei [area alpinus].

49. Malcolmia R.Br. 106. Malcolmia angulifolia [2000 m.].

50. Barbarea R. 107. Barbarea bracteosa [1300-2000 m.]; 108. Barbarea balcana [1300-2000 m.].

51. Roripa Scop.109. Roripa pyrenaica [Kodra e diellit (Popova Shapka) Cerepashina, Luboten 600-2.500 m.].

52. Cardamine L. 110. Cardamine bulbifera (L.) [Jellak, Leshnica]; 111. Cardamine acris Griseb.[Kodra e diellit (Popova Shapka), Cerepashin, Luboten 1200-2.650 m.]; 112. Cardamine glauca Sprengel[Kobilica 1000-2600 m.]; 113. Cardamine impatiens (L.) [Leshnica, Luboten 5.50-1.700 m.]; 114. Cardamine flexuosa With. [River Luboten]. 53. Arabis L. 115. Arabis sagittata (Bertol.) [to 2000m.]; 116. Arabis allionii [1500-2700 m.]; 117. Arabis muralis Bertol.[2100 m.]; 118. Arabis turrita L.[1.800 m.]; 119. Arabis alpine L.[2700 m.]; 120. Arabis glabra L.[200-2000 m.].

54. Aubrieta Adanson 121. Aubrieta scardica (Wettst.)[2.700 m.]; 122. Aubrieta gracilis Spruner ex Boiss. [Luboten, area alpine and subalp.].

55. Lunaria L. 122. Lunaria rediviva [2000 m.].

56.Alyssum L. 123. Alyssum scardicum [2000-2.764 m.].

57.Ptilotrichum C. A. Meyer 124. Ptilotrichum rupestre [Luboten, Kobilica].

58. Draba L. [1.500-2.700 m.]; 125. Draba lasiocarpa Rochel, Sched, Pl, Hung. [Luboten]; 126. Draba lacaitae Boiss., Fl. Or.[Cerepashina, 2.500 m.]; 127. Draba doerfleri Wettst. [Ripin alpik]; 128. Draba muralis L., Sp., Pl., [1.700 m.].

59. Thlaspi L. 129. Thlaspi microphyllum Boiss. [2.700 m.]; 130. Thlaspi praecox Wulfen in Jack. [2.700 m.]; 131. Thlaspi bellidifolium Grisch., [2.700 m.].

60. Iberis L.132. Iberis sempervirens L., Sp., Pl. [1.000-2.300 m.].

21. Salicaceae

61. Populus L. 133. Populus tremula L. [2.000].

62. Salix L. 134. Salix reticulate L.[Vende me gurishta, on limestone rocky 2000-2300 m.]; 135. Salix retusa L., Syst.,Nat. [Në shkëmbinj dhe plasaritje të shkëmbinjve. 1900-2500 m. Alpike]; 136. Salix alpina Scop. [1700-2700 m.]; 137. Salix silesiaca Wild.,Sp., Pl. [Ellak,Leshnica, 1.650 m.]; 138. Salix caprea L., Sp., Pl.[2000 m.]; 139. Salix waldsteiniana Wild. Sp. Pl. [1700 m. Trip for Leshnica].

22. Ericaceae

63. Bruckenthalia Reichenb. 140. Bruckenthalia spiculifolia [Heats, alpine pastures, on silicate substrate or deep soil usually in subalpine belt].

64. Rhododendron L. 141. Rhododendron ferrugineum L., Sp. Pl. [Stony slopes near Livadica Lake 2200-2400m.].

65. Arctostaphylos Adanson. 142. Arctostaphylos uva ursi (L) Sprengel, Syst. [area subalpine mbi gurishta gëlqeror. $1650-2200 \mathrm{~m}$.$] .$

66. Vaccinium L. 143. Vaccinium myrtillus. L. Sp. Pl. [Heats, alpine pastures 1000-2200 m. Cosm.].

23. Pyrolaceae

67. Pyrola L. 144. Pyrola minor L. [1900 m.]. 145. Pyrola media Swartz, Kungl. Svenska Vet. [1900 m.].

68. Moneses Salisb. 146. Moneses uniflora (L.) A. Gray. Man. Bot. [1.400-2.000 m.].

24. Monotropace

69. Monotropa L. 147. Monotropa hypopitys L., Sp. Pl. [1000-2000 m.].

25. Empetraceae

70. Empetrum L. 148. Empetrum nigrum L., Sp. Pl. [area alpine and subalpine.].

26. Primulaceae,

71. Primula L. 149. Primula elatior (L.) Hill, Veg. Syst. [Sharr mountain 1.700-2.700 m.]; 150. Primula veris L. Sp. Pl. [2.400 m.]; 151. Primula halleri Gmelin, Onomat. Bot. Compl.[Sharr mountain 1.600-2.700 m.]; 152. Primula minima L., Sp. Pl.

72. Androsaceae L. 153. Androsacea hedraeantha Griseb., Spic. Fl. Rumel.[Nëpër barishte deri 2700 m.]. 154. Androsaceae villosa L. Sp. Pl.[1800-2700 m.];

73. Soldanella L. 155. Soldanela pindicola Hausskn., Mittel. Georg. Ges. Jena. [mbi 1.500 m.]; 156. Soldanela macedonica Meyer F. K. [mbi 1.700 m.].

27. Tiliaceae

74. Tilia L. 157. Tilia platyphyllos Scop., Fl. Carn. [Luboten, Leshnica, deri 1.650 m.]. 


\section{Malvaceae}

75. Malva L.158. Malva moschata [deri $2000 \mathrm{~m}$.]; 159. Malva neglecta [deri $2.200 \mathrm{~m}$.].

\section{Euphorbiaceae}

76. Euphorbia L. 160. Euphorbia stricta L. [to $2.000 \mathrm{~m}$. Cerepasina]; 161. Euphorbia cyparissias L., Sp. Pl. [to 2.200m.]; 162. Euphorbia epithymoides [Ellak, Leshnica].

30. Thymelaceae

77. Daphne L. 164. Daphne mezereum L. [1200.2300 m.]; 165. Daphne oleoides L., [1200-2000 m.].

\section{Rosaceae}

78. Aruncus Adans. 166. Aruncus vulgaris Rafin., Sylva Tellur. [for 1.000-1.700 m.].

79. Rubus L. 167. Rubus idaeus L., Sp. Pl. [1200-2000 m.].

80. Rosa L. 168. Rosa canina L. [Sharr Mountain]; 169. Rosa glauca Pourret. [1200-2000 m.]; 170. Rosa pindulina L., Sp., Pl. [1200-2000 m.]; 171. Rosa dumalis Sharr mountain]; 172. Rosa corymbifera Borkh., Vers. Forstbot Beschr. Holzart.[Sharr mountain]; 173. Rosa tomentosa Sm., Fl. Brit.[300-2300 m.]; 174. Rosa villosa L. 10001.600 m.]; 175. Rosa orientalis Dupont ex Ser. In., DC., Prodr. [1.500-2000 m.];

81. Sanguisorba L.176. Sanguisorba officinalis L. [1200-2500 m.]; 177. Sanguisorba minor L., Sp. Pl. [to 2000 m.]. 82. Dryas L.178. Dryas octopetala [1700-2500 m.].

83. Geum L. 179. Geum reptans [to 2750 m.]; 180. Geum urbanum [1600 m.]; 181. Geum montanum L. [to 2760 m.]; 182. Geum rivale L.[1500-2400 m.]; 183. Geum coccineum L. [1000-2300 m.].

84. Potentilla L. 184. Potentilla argentea L.[2000 m.]; 185. Potentilla inclinata Vill., Hist. Pl. Dauph. [to 2000 m.]; 186. Potentilla astracanica Jacq., Misc. Austr. Bot. [1.600 m.]; 187. Potentilla pedata Nestler, Monogr. Potent. [to 2.000 m.]; 188. Potentilla obscura Wild., Sp. Pl. Ed. [to 2200 m.]; 189. Potentilla sulphurea Lam., Fr. Ed. [1.800 m.]; 190. Potentilla balcanica Wolf Micevski [to 2.200 m.]; 191. Potentilla aurea L. [1.600-2.700 m.]; 192. Potentilla speciose Willd., Sp. Pl. [1400-2100 m.].

85. Fragaria L. 193. Fragaria vesca [to 2000 m.]; 194. Fragaria moschata L. [Sharr Mountain].

86. Alchemilla L. 195. Alchemilla acutata Buser, Algem. Bot. Zeit. [2.450 m.]; 196. Alchemilla bulgarica Rothm. Feddes Repet [2.370 m.]; 197. Alchemilla flabellate Buser, Not. Alchim [-2.500 m. Kobilica, Luboten]; 198. Alchemilla glaucescens Wallr., Linnaea [1.950 m. Luboten]; 199. Alchemilla pirinica Pawl., Bull. Int. Acad. Polon. [2.662 m.Rudoka, Cerepashina, Luboten, Leshnica]; 200. Alchemilla serbica (Fritsch) Pawl., Acta Bot. Pol.[to2.120 m. Cerepashina]; 201. Alchemilla acutiloba Opiz, Fl. Bohm [2.120 m. Gajre, Jellak, Cerepashina]; 202. Alchemilla crinite Buser, Scrin. Fl. Select. Magnier [to 2.320 m. Cerepashina, Kobilica]; 203. Alchemilla gracilis Opiz, Fl. Bohm [to 1.890 m. Gajre, Kodra e diellit (Popova Sapka)]; 204. Alchemilla heterophylla Rothm., Feddes Repert. [to $2.520 \mathrm{~m}$. Leshnica, Cerepashina]; 205. Alchemilla monticolla Opiz, Fl. Bohm[to 2.560 m. Plloça-Sharr mountain]; 206. Alchemilla xanthochlora Rothm., Feddes Repert. [to 2.330 m.]; 207. Alchemilla connivens Buser, Bull. Herb. Boiss. [Majen e Lubotenit 2.300 m.]; 208. Alchemilla effuse Buser Alchim Walais [to 2000 m.]; 209. Alchemilla reniformis Buser Alchim Walais [to $2.400 \mathrm{~m}$.]; 210. Alchemilla heterotricha Roth; Feddes Repert. [to 2.200 m. Kobilica, Luboten]; 211. Alchemilla fissa Gunther \& Schumnel, Sched. Cend. Siles. Exsicc. [to 2200 m. Luboten].

87. Pyrus L. 212. Pyrus pyraster Burgsd., Anleit Holzart. [to $1.600 \mathrm{~m}$ Sharr mountain].

88. Malus Miller 213. Malus sylvestris Miller, Gard. Dict. Ed [to 1.600 m.].

89. Sorbus L. 214. Sorbus semipinnata (Roth. Hedlund, Monogr.Gatt. Sorbus [1200-1850 m. Cerepashina, Leshnica]; 215. Sorbus chamaemespillus L. [area subalpine, Sharr mountain].

90. Amelanchier Medicus 216. Amelanchier ovalis Medicus [2.100 m. Sharr mountain].

91. Cotoneaster Medicus 217. Cotoneaster integerrimus Medicus [to 2.200 m.Sharr mountain]. 218. Cotoneaster nebrodensis (Guss.) [to $2000 \mathrm{~m}$. Sharr mountain].

92. Crataegus L. 219. Crataegus sericeus Dzhekov [1.000-1.600m.].

93. Prunus L. 220. Prunus cerasifera Ehrh., Beitr. Naturk. [to 1.750 m.]. 221. Prunus cocomilia Ten., Flnap [Luboten to $1.750 \mathrm{~m}$.]; 222. Prunus avium L. [1.800 m.].

\section{Grossulariaceae}

94. Ribes L. 223. Ribes uva-crispa [1.200-1.800 m].

33. Crassulaceae

95. Sempervivum L. 224. Sempervivum thompsonianum Wale, Quart. Bull. Alp. Gard. Soc. [2.100-2.200 m.]; 225. Sempervivum kosaninii Praeger Inst. Univ. Beograd [Sharr mountain]; 226. Sempervivum marmoreum Griseb. Spicil. Fl. Rumel. [Sharr mountain].

96. Sedum L. 227. Sedum acre L. [2.300 m.]. 228. Sedum sartorianum Boiss., Diagn. Pl. Or. Nov. [alpike, subalpike, to 1.800 m.]; 229. Sedum alpestre Wild., Prosp. Pl. Dauph. [Sharr mountain]; 230. Sedum erythraeum Griseb. Spicil. Fl. Rumel[2.000 m.]; 231. Sedum flexuosum Wettst. [Sharr mountain]; 232. Sedum album L. [to 2.300 m.]; 233. Sedum dasyphyllium L [to 2.500 m.]; 234. Sedum magelense Ten., Prodr. Fl. Nap. [1.000-2.500 m.]; 
235. Sedum atratum L. [2.500 m.]; 236. Sedum annuum L. [2.500 m. Sharr Mountain]; 237. Sedum hispanicum L. [1.800 m.].

\section{Saxifragaceae}

97. Saxifraga L. 238. Saxifraga rotundifolia L., Sp. Pl. [to 2.700 m.]; 239. Saxifraga bryoides L., Sp. Pl. [2.2002.600 m.]; 240. Saxifraga tridactylites L., Sp. Pl. [to 1.700 m.]; 241. Saxifraga adscendens L., Sp. Pl. [area alpine]; 242. Saxifraga aizoides L., Sp. Pl. [Luboten]; 243. Saxifraga glabella Bertol., Gior. Arcan. Sci. [Sharr mountain]; 244. Saxifraga pedemontana Al., Fl. Peden [Sharr Mountain, Maja e zezë (Crn Vrv) Sharr mountain]; 245. Saxifraga androsaceae L., Sp. Pl. [Sharr mountain 1.700-2.500 m.]; 246. Saxifraga bulbifera 1700 m.]; 247. Saxifraga exarata Wild., Prosp.Pl. Dauph [Sharr mountain, alpines area]; 248. Saxifraga bulbifera L. Sp. Pl. [to 1.700-1.800 m.]; 249. Saxifrage opposipifolia L., Sp. Pl. [alpines area]; 250. Saxifraga marginata Sternb., Revis. Saxifr. Su. Pl [alpinus and subalpinus area]; 251. Saxifraga scardica Griseb. Spicil. Fl. Rumel [Sharr mountain]. 252. Saxifraga paniculata Miller [400-2.700 m.];

98. Chrysosplenium L. 253. Chrysosplenium alternifolium [Sharr Mountain].

35. Leguminosae

99. Cytisus L. 254. Cytisus nigricans Sp. Pl. [Luboten].

100. Chamaecytisus Link. 255. Chamaecytisus triflorius (Link) [2.000 m.].

101.Genista L. 256. Genista depressa M. B., Fl. Taur.-Caucas. [2.700 m. Sharr mountain].

102. Astragallus L. 257. Astragallus depresus [700-2.400 m. R. M.]; 258. Astragallus australis (L.) Lam., Fl. Fr. [2.200-2.650 m.].

103. Oxytropis DC. 259. Oxytropis lapponica (Wahlenb.) Gay, Flora [Cerepashin,Bargdan, Maja e diellit (Popova Sapka) 2.000-2.7600 m.]; 260. Oxytropis campestris (L) DC., Astrag. [1.560-2.400m. Cerepashin, Luboten]; 261. Oxytropis dinarica (Murb.) Wettst., Bibl. Bot. [1.700-2.650 m. Kodra e diellit (Popova Sapka), Ellak, Cerepasihin, Maja e Diellit]; 262. Oxytropis halleri Bunge ex Koch [Maja e Diellit (Maja e Titos)].

104. Vicia L. 263. Vicia icana Gouan, Fl. Monspl. [to 2.300 m.]; 264. Vicia sylvatica L. Sp. Pl. [800-2.000 m., Leshnica]; 265. Vicia tetrasperma (L.) Schreb ., Spic. [to 1.600 m. Rogaçeva]; 266. Vicia sepium L. Sp. Pl. [7001.750 m.]; 267. Vicia lathyroides L. Sp. Pl. [to $1.600 \mathrm{~m}$.];

105. Lathyrus L. 268. Lathyrus laxiflorus (Desf.) O. Kuntze, Acta Horti Petrop. [to 1.600 m.]; 269. Lathyrus Nissolia L., Sp. Pl.[to 1.700 m.];

106. Trifolium L., Sp. Pl. 270. Trifolium repens L., Sp. Pl. [to alpines area]; 271. Trifolium pallescens Schreber in Sturm, Deutschl. Fl. Ab. L, Bd. 4 H. [mbi 2.000 m.]; 272. Trifolium badium Schreber in Sturm, Deutschl. Fl. Ab. L, Bd. 4 H. 273. Trifolium velenovskyi Vand., Sitz.-Ber. Bohm. Ges. Wiss. (Math.-Nat. Kl.) [1.000-2.000 m. Sh.M.]; 274. Trifolium noricum Wulfen, Arch. Bot. (Roemer) [1.650-2.700 m.-endemike]; 275. Trifolium medium L., Amoen. Acad. [700-2.100 m. R.M.]; 276. Trifolium pignantii Fauche \& Chaub. In Bory. Exped. Sci. Moree [to 2.000 m.]; 277. Trifolium alpestre L., Sp. Pl. ed. [to 2.000 m.]; 278. Trifolium ochroleucon Hudson, Fl. Angl. [to $1.700 \mathrm{~m}$.$] .$

107. Dorycnium Miller 279. Dorycnium herbaceum Will., Prosp. Pl. Dauph. [Luboten]; 280. Dorycnium hirsutum L. 108. Lotus L. 281. Lotus corniculatus L. var alpinus Ser. In Dc., Prodr. [alpinus area-Kodra e diellit (Popova Sapka)].

109. Anthyllis L. 282. Anthyllis aurea Welden in Host, Fl. Aust. [700-2.000 m. Kodra e Diellit (Popova Sapka) Cerepashina]; 283. Anthyllis vulneraria L. SP. Pl. var. albana [Kodra e Diellit (Popova Sapka) Cerepashina 2.50$2.650 \mathrm{~m}$.$] .$

110. Hippocrepis L. 284. Hippocrepis comosa L. SP. Pl. [2.200 m. Sharr Mountain].

111. Onobrychis Miller 285. Onobrychis montana Dc.in Lam. Et Dc., Fl. Fr. ed. subsp. scardica (Griseb) Ball, Feddes repert.[Sharr Mountain].

36. Onagraceae

112. Circea L. 286. Circea lutetiana L. SP. Pl. [2.000 m. Luboten].

113. Epilobium L. 287. Epilobium angustifolium L. SP. Pl. [Rocky grounds; waste areas; mountain paths; forest edges and cuttings]; 288. Epilobium parviflorum Screb., Spicil, Fl. Lips. [Sharr Mountain-Vejce]; 289. Epilobium montanum L., Sp. Pl. [Sharr Mountain]; 290. Epilobium collinum Gmelin, Fl. Bad. [Sharr Mountain]; 291. Epilobium lanceolatum Sebastini \& Mauri, Fl. Rom. [Sharr Mountain to 1.600 m.]; 292. Epilobium obscurum Schreber, Spic. [Sharr Mountain-Luboten].

\section{Aceracea}

114. Acer L. 293. Acer platanoides L. SP. Pl. [Mali Sharr-Luboten, LeshnicA]; 294. Acer campestre L. SP. Pl.; 295. Acer pseudoplatanus L. SP. Pl. [900.-1.700 m.Tetova-Lisec]; 296. Acer heldreichii Orph. In Boiss.; Diagn. Pl. Orient. [1.400-2.000 m. Leshnica, Kodra e Dielli, Lisec]; 297. Acer obtusatum Waldst. \& Kit. In Wild., Sp. Pl. [600- 
1.300 (1700 m.)]; 298. Acer hyrcanum Fischer \& C. A. Meyer, Ind. Sem. Hort. Petrop. [to 1.600 m.]; 299. Acer monspessulanum L. SP. Pl. [Tetova-Lisec].

38. Linaceae

115. Linum. 300. Linum perenne L. SP. Pl. subsp. extraaxillare [to 1.800 m.]; 301. Linum catharticum L. SP. Pl. [to $2.200 \mathrm{~m}$.$] .$

39.oxalidaceae

299. Oxalis acetosalla L. SP. Pl. [Sharr mountain].

40. Geraniaceae

116. Geranium L. 302. Geranium macrorrhizum L. SP. P1. [Sharr Mountain]; 303. Geranium cinereum Cav., Monad. Class. Diss. Dec. subsp. subcaulescens [for 1.200-2.700 m.]; 304. Geranium sylvaticum L. SP. Pl. [to 2.100 m. Sharr montain]; 305. Geranium reflexum L., Mantissa Alt. [Sharr mountain1.800 m.]; 306. Geranium aristatum Freyn \& Sint., Bull. Herb. Boiss [1.000-2.650 m. Sharr Mountain]; 307. Geranium pyrenaiucum Burm. Fil., Spec. Bot. Geran. [to $2.200 \mathrm{~m}$. Sharr Mountain]; 308. Geranium molle L. SP. Pl. [Sharr Mountain]; 309. Geranium robertianum L. SP. Pl. [2.000 m. Sh.M.].

\section{Polygalaceae}

117. Polygala L. 310. Polygala major Jack., Fl. Austr. [2.000 m. Rogaçeva, Sharr Mountain]; 311. Polygala comosa Schkuhr, Handb. [1.800 m. Kodra e Diellit, Luboten-1.800 m.]; 312. Polygala vulgaris L. SP. Pl. [Sharr mountain, to 2.500 m.]; 313. Polygala alpestris Reichenb., Ic. Bt Pl. Crit [to 2.000 m.].

42. Umbelliferae

118. Sanicula L. 314. Sanicula europaea L., Sp. Pl. [to 1.800 m.].

119. Astrantia L. 315. Astrantia major L., Sp. Pl. [Kodra e Diellit-Leshnica].

120. Chaerophyllium L. 316. Chaerophyllum hirsutum L., Sp. Pl. [Sharr Mountain]; 317. Chaerophyllum aureum L. L., Sp. Pl. [Sharr Mountain-Luboten, to $2.000 \mathrm{~m}$.].

121. Huetia Boiss 318. Huetia Cynapioides (Guss.) P.W.Wall, Feddes Repert. [to 2.000 m.].

122. Pimpinella L. 319. Pimpinella major (L.) Hudson, Fl. Angl. [900-1.700 m.]; 320. Pimpinella saxifraga L., Sp. Pl. [1.400-2.200 m. Sharr Mountain-Luboten].

123. Aegopodium L. 321. Aegopodium sp. L., Sp. Pl. [to 1.800 (2.000 m.)];

124. Seseli L. 322. Seseli peucedanoides (Bieb.) Kos.-Pol., Bull. Soc. Nat. Moscou, Nova ser. [to 2.200 m.].

125. Athamanta L. 323. Athamanta haynaldi Borb. \& Uechtr. Bot. Zeitschr. [to 2.000 m.].

126. Meum Miller 324. Meum athamanticum Jack., Fl. Austr. [1.500-2.500 m.].

127. Bupleurum L. 325. Bupleurum ranunculoides L., Sp. Pl. [Sharr Mountain-Luboten]; 326. Bupleurum falcatum L., Sp. Pl. [to $2.100 \mathrm{~m}$.$] .$

128. Trinia Hoffm. 327. Trinia glauca (L.) Dumort., Fl. Belg. subsp. carniolica [Cerepashina, Ellak, Luboten, Maja e Diellit, Kodra e Diellit]; 328. Trinia dalechampii (Ten.) Janchen, Oster. Bot. Zeitschr. [Sharr Mountain-Luboten].

129. Carum L. 329. Carum multiflorum (Sm) Boiss., Fl. Or.[to 2.000 m.];

130. Cnidium Cusson 330. Cnidium silaifolium (Jack.) Simonkai, Enum. Fl. Transs. [to 1.700 m.].

131. Peucedanum L. 331. Peucedanum oligophyllum (Griseb.) Vandas, Magyar Bot. Lapok [Sharr Mountain, 1.500 m.]; 332. Peucedanum aegopodioides (Boiss.) Vandas, Sitz. Ber. Bohm. Ges. Wiss. (Math.- Nat. Kl.). [Luboten].

132. Pastinaca L. 333. Pastinaca sativa L., Sp. Pl. subsp. urens [Lisec].

133. Heracleum L. 334. Heracleum sphondylium L., L., Sp. Pl. subsp. pyrenaicum (Lam.) Bonnier \& Layens, Fl. Fr. [Luboten].

43. Celastraceae

134. Evonymus L. 335. Evonymus latifolius (L.) Miller, Gard. Dict. [Sharr Mountain, Leshnica, Luboten].

44. Rhamnaceae

135. Rhamnus L. 336. Rhamnus alpine L., L., Sp. Pl. subsp. fallax (Boiss.,) Maire \& Petitmenegin, Bull. Soc. Sci. Nancy [Sharr Mountain]; 337. Rhamnus pumila Turra, Giorn. Ital. Sci. Agric. Arti Commerc [Lisec, Kodra e Diellit, Cerepashin, Ellak, Leshnica].

45. Santalaceae

136. Thesium L. 338. Thesium parnassi A. DC., Prodr. [Cerepashina, Bargdan, Leshnica, Luboten,]; 339. Thesium alpinum L., Sp. Pl. [1.600-2.000 m., Sharr Mountain-Kodra e Diellit, Ellak, Leshnica].

46. Caprifoliaceae

137. Knautia L. 340. Knautia arvensis [Sharr Mountain].

138. Sambucus L. 341. Sambucus nigra L. [Sharr Mountain]; 342. Sambucus ebulus L. [Sharr Mountain]; 343. Sambucus racemose [deciduous and coniferous forests; shrubby areas, in ravines- Ellak, tre ujëra (Tri vodi) 14001900 m.].

47. Gentianaceae 
139. Centaurium Hill 344. Centaurium erythraea Rafn, Danm. Holst. [Sharr Mountain-Luboten].

140. Gentiana L. 345. Gentiana lutea L., Sp. Pl. [1.300-2.200 m.]; 346. Gentiana punctate L., Sp. Pl. [1.800-2.550 m. Sharr Mountain- Vraca]. 347. Gentiana asclepiadea L., Sp. Pl. [for 1200-2000 m.]; 348. Gentiana cruciata L., Sp. Pl. [700-1700 m.]; 349. Gentiana verna L., Sp. Pl. subsp. balcanica Pritchard., [1500-2700 m.]; 350. Gentiana utriculosa L., Sp. Pl. [Sharr Mountain].

141. Gentianella Moench. 351. Gentianella ciliate (L.) Borkh., Arch. Bot. (Roemer), [Sharr Mountain-Kodra e Diellit]; 352. Gentianella bulgarica (Vel.) J. Holub, folia geobot, Phytotax. (Praha) [Sharr Mountain- Kodra e Diellit (Popova Sapka)].

\section{Convolvulaceae}

142. Cuscuta L 353. Cuscuta approximate Bab., Ann. Mag. Nat. Hist. [Sharr Mountain-Kobilica].

143. Calystegia R. Br. 354. Calystegia sylvatica (Kit.) Griseb., Spic. Fl. Rumel. [Sharr Mountain].

49. Boraginaceae

144. Cerinthe L. 355. Cerinthe glabra Miller, Gard. Dict. ed. [Ellak-Leshnica 1600-2650 m.].

145. Alkanna Tausch 356. Alkana scardica [Ellak-Leshnica, Kobilica, 1600-1900 m.].

146. Echium L. 357. Echium vulgare L., Sp. Pl. [Tetova-Lisec, to 1700 m.].

147. Symphytum L. 358. Symphytum tuberosum L., Sp. Pl. subsp. angustifolia (A. Kerner) [Lisec-Kodra e Diellit (Popova Sapka) to $2250 \mathrm{~m}$ ].

148. Anchusa L. 359. Anchusa officinalis L., Sp. Pl. [Tetovë-Lisec, to 1700 m.]; 360. Anchusa cretica [M. Sh. to area alpine].

149. Myosoitis L. 361. Myosoitis stricta [Link ex Roemer \& Schultes, Syst, Veg. [for 200-1700 m. Lisec-Kodra e Diellit]; 362. Myosoitis sylvatica Hoffm., Deutschl. Fl. [for 700-2650 m. Luboten]; 363. Myosoitis suaveolens Wald. \& Kit. Ex Wild., Enum. Pl. Hort. Berol. [Luboten, (1000) 1600-2600 m.]; 364. Myositis scorpioides L., Sp. Pl. [ Sharr Mountain-Lisec, Kodra e Diellit].

150. Cynoglossum L. 365. Cynoglossum sp. L., Sp. Pl. [to 1800 m. Sharr Mountain-Lisec, Kodra e Diellit].

50. Scrophulariaceae

151. Linaria L. 366. Linaria vulgaris Miller [Buzë pyjesh me ah].

152. Digitalis L. 367. Digitalis ferruginea L. [Sharr Mountain]; 368. Digitalis grandiflora L. [Sharr Mountain].

51. Plantaginaceae

153. Plantago L. 369. Plantago major L. [Sharr Mountain]; 370. Plantago lanceolate L. [Sharr Mountain]; 371. Plantago media L. [Sharr Mountain].

52. Lamiaceae

154. Sideritis 372. Sideriti scardica [Luboten, 1200-2200 m.].

155. Thymus 373. Thymus striatus Vahl. [Sharr Mountain].

53. Campanulaceae

156. Edraianthus A. DC. 374. Edraianthus graminiflorus Dc. [Luboten, 1500-2300 m.].

54.

ASTERACEAE (COMPOSITAE)

157. Erigeron L. 375. Erigeron alpinus [ Sharr mountain].

158. Matricaria L. 376. Matricaria chamomilla [Sharr Mountain].

159. Bellis L. 377. Bellis annua L. [ Sharr mountain].

160. Leucantemum Mill. 378. Leucanthemum vulgare [Sharr Mountain].

161. Cichorium 379. Cichorium intybus [Shar Mountain].

162. Achillea L. 380. Achillea clypeolata [Sharr Mountain]; 381. Achillea millefolium L. [Shar Mountain].

55. Amaryllidaceae

163. Galanthus L. 382. Galanthus nivalis L. [area subalpina].

56. Xanthorrhoaceae

164. Asphodelus L. 383 . Asphodelus albus Wild. [area subalpina]

57. Iridaceae

165. Crocus L. 384. Crocus bulbs L. [Kodra e Diellit (Popova Shapka), 1750 m.]; 385. Crocus scardicus Kosanin [area alpina, 1800-2500 m.]. 386. Crocus chrysanthus Herb., 387. Crocus vernus (L.) Hill. [area subalpina].

58. Liliaceae

166. Fritillaria L. 388. Fritillaria macedonica Bornm. [Bogovina's lake];

167. Lilium L. 389. Lilium albanicum Griseb. [Bogovina's lake]; 390. Lilium martagon L. [Sharr MountainLuboten, area subalpine].

59. Asparagaceae

169. Scilla 392. Scilla bifolia L. [Sharr Mountain- Kodra e Diellit (Popova Sapka)].

60. Allioideae 
170. Allium L. 393. Allium roseum L. [Bogovia's Lake, area alpina].

61. Juncaceae

171. Juncus L. 394. Juncus effuses L. [Luboten, area subalpina]

62. Melanthiaceae

172. Veratrum L. 395. Veratrum album L. [area alpina]; 396. Veratrum nigrum L. [area alpina];

173. Narthecium Huds 397. Narthecium scardicum Kosanini [Xhinbeg, area alpina].

Table number. 2. The total number of families, genus and species in Sharr Mountain

\begin{tabular}{|c|c|c|c|}
\hline No & families & genus & species \\
\hline 1. & LYCOPODIACEAE & 2 & 2 \\
\hline 2. & OPHYOGLOSSACEAE & 1 & 1 \\
\hline 3. & CRYPTOGRAMMACEAE & 1 & 1 \\
\hline 4. & ASPLENIACEAE & 1 & 2 \\
\hline 5. & ATHYRIACEAE & 1 & 1 \\
\hline 6. & PINACEAE & 3 & 4 \\
\hline 7. & CUPRESACEAE & 1 & 2 \\
\hline 8. & TAXACEAE & 1 & 1 \\
\hline 9. & RANUNCULACEAE & 8 & 16 \\
\hline 10. & FUMARIACEAE & 1 & 1 \\
\hline 11. & URTICACEAE & 1 & 2 \\
\hline 12. & FAGACEAE & 1 & 1 \\
\hline 13. & BETULLACEAE & 2 & 2 \\
\hline 14. & CARYOPHYLLACE & 15 & 34 \\
\hline 15. & POLYGONACEAE & 3 & 10 \\
\hline 16. & PLUMBAGINACEAE & 1 & 2 \\
\hline 17. & HYPERICACEAE & 1 & 3 \\
\hline 18. & VIOLACEAE & 1 & 12 \\
\hline 19. & CISTACEAE & 1 & 3 \\
\hline 20. & CRUCIFERAE & 14 & 32 \\
\hline 21. & SALICACEAE & 2 & 7 \\
\hline 22. & ERICACEAE & 4 & 4 \\
\hline 23. & PYROLACEAE & 2 & 3 \\
\hline 24. & MONOTROPACE & 1 & 1 \\
\hline 25. & EMPETRACEAE & 1 & 1 \\
\hline 26. & PRIMULACEAE & 3 & 8 \\
\hline 27. & TILIACEAE & 1 & 1 \\
\hline 28. & MALVACEAE & 1 & 1 \\
\hline 29. & EUPHORBIACEAE & 1 & 3 \\
\hline 30. & THYMELACEAE & 1 & 2 \\
\hline 31. & ROSACEAE & 16 & 57 \\
\hline 32. & GROSSULARIACEAE & 1 & 1 \\
\hline 33. & CRASSULACEAE & 2 & 14 \\
\hline 34. & SAXIFRAGACEAE & 2 & 16 \\
\hline 35. & LEGUMINOSAE & 13 & 31 \\
\hline 36. & ONAGRACEAE & 2 & 7 \\
\hline 37. & ACERACEA & 1 & 7 \\
\hline 38. & LINACEAE & 1 & 2 \\
\hline 39. & OXALIDACEAE & 1 & 1 \\
\hline 40. & GERANIACEAE & 1 & 8 \\
\hline 41. & POLYGALACEAE & 16 & 4 \\
\hline 42. & UMBELLIFERAE & 1 & 21 \\
\hline 43. & CELASTRACEAE & 1 & 1 \\
\hline 44. & RHAMNACEAE & 1 & 2 \\
\hline
\end{tabular}




\begin{tabular}{|l|l|c|c|}
\hline 45. & SANTALACEAE & 2 & 2 \\
\hline 46. & CAPRIFOLIACEAE & 3 & 4 \\
\hline 47. & GENTIANACEAE & 2 & 9 \\
\hline 48. & CONVOLVULACEAE & 7 & 11 \\
\hline 49. & BORAGINACEAE & 1 & 3 \\
\hline 50. & SCROPHULARIACEAE & 1 & 3 \\
\hline 51. & PLANTAGINACEAE & 1 & 2 \\
\hline 52. & LAMIACEAE & 6 & 1 \\
\hline 53. & CAMPANULACEAE & 6 & 7 \\
\hline 54. & ASTERACEAE (COMPOSITAE & 1 & 1 \\
\hline 55. & AMARYLLIDACEAE & 1 & 4 \\
\hline 56. & XANTHORRHOACEAE & 1 & 3 \\
\hline 57. & IRIDACEAE & 2 & 1 \\
\hline 58. & LILIACEAE & 1 & 1 \\
\hline 59. & MELANTHIACEAE & 1 & 1 \\
\hline 60. & ASPARAGACEAE & 1 & 1 \\
\hline 61. & ALLIOIDEAE & 1 & 3 \\
\hline 62. & JUNCACEAE & 2 & 397 species \\
\hline 63. & MELANTHIACEAE & 173 genus \\
\hline & Total: families $=63$ & & 1 \\
\hline
\end{tabular}

\section{Conclusion:-}

In editions of domestic and foreign authors, it is said that there are about 2000 species in the flora of Sharr Mountain.

The current partial processing of collected materials consists of 590 species, belonging to 216 genus and 63 families. These data testify a rich flora of the massif of Sharr.

By analyzing and comparing our current material with what is known to date for Macedonia, it is noticed a significant similarity and high numbers of endemic plants.

Wide Areal have family species in all heights of Shara: Ranunculaceae, Caryophyllaceae, Crucifer, Rosaceae, Leguminosae, Umbelliferae.

Also it is noted the used literature that there is no data on family "plants and plants Composite, Monocotyledonae" and a large number of families with a smaller number of plants.

Further elaboration of material of flora in Shara in the study will fill the number of species prevalent in flora in the Shara.

\section{References:-}

1. Haziri, A. (2012) FITOCENOLOGJIA FLORA DHE VEGJETACIONI I MAQEDONISË (Dispensë).

2. Shaban Shehapi. Tetovë 2015. Punim diplome „Karakteristikat fiziko- gjografike të Malit Sharr”

3. GEOLOGICAL ASPECTS OF THE RESEARCHED REGION (Менковић, 1978).

4. Skenderi, F.(2009) Klimatologjia, Tetovë

5. Mustafa Demiri, (1983) „FLORA EKSKURSIONISTE E SHQIPËRISË” Tiranë: Mihail Duri.

6. Micevski K. (1985-2005): Flora na Republika Makedonija. 1-6, MACEDONIA ACADEMY OF SCIENCES AND ARTS. Skopje.

7. TEACHING PRACTICES IN BOTANY "Murat Xhulaj, Tirana 2015 which was used to identify the species.

8. Kokaljari, P. Z. (2015). Bimët mjekësore në familje. Tirana.

9. Melovska, N. (2015). Field guidebook to mountain flowers Sharr Planina. Skopje 